\title{
Future directions of positron annihilation spectroscopy in low-k dielectric films
}

\author{
D. W. Gidley ${ }^{*}$, R. S. Vallery, M. Liu, and H.-G. Peng \\ Department of Physics, University of Michigan, Ann Arbor, MI 48109, USA
}

Received 31 August 2006, accepted 1 December 2006

Published online 16 July 2007

PACS 36.10.Dr, 77.55.+f, 78.55.Mb, 78.70.Bj

Positronium Annihilation Lifetime Spectroscopy (PALS) has become recognized in the microelectronics industry as one of only several methods capable of quantitatively characterizing engineered nanopores in next-generation $(\mathrm{k}<2.2)$ interlayer dielectric (ILD) thin films. Successes and shortcomings of PALS to date will be assessed and compared with other methods of porosimetry such as ellipsometric and X-ray porosimetries (EP and XRP). A major theme in future low-k research focuses on the ability to integrate porous ILD's into chip fabrication; the vulnerability of porous dielectrics to etching, ashing, and chemical-mechanical polishing in process integration is delaying the introduction of ultra-low-k films. As device size approaches $45 \mathrm{~nm}$ the need to probe very small (sub-nanometer), semi-isolated pores beneath thin diffusion barriers is even more challenging. Depth-profiled PALS with its ability to determine a quantitative pore interconnection length and easily resolve $0.3 \mathrm{~nm}$ pores beneath diffusion barriers or in trench-patterned dielectrics should have a bright future in porous ILD research. The ability of PALS (and PAS in general) to deduce evolution and growth of pores with porosity should find broad applicability in the emerging field of high performance materials with strategically engineered nanopores.

(C) 2007 WILEY-VCH Verlag GmbH \& Co. KGaA, Weinheim

1 Introduction The embracing of positron annihilation spectroscopy (PAS) by the microelectronics industry to characterize pores strategically engineered into low-k interlayer dielectrics continues to be one of the most successful industrial applications of PAS [1]. Much of this success stems from the dearth of more traditional techniques to probe pores at the nanometer size scale in thin $(\sim 300 \mathrm{~nm})$ films of amorphous insulators. The thrust of this paper is to review some recent PAS progress and assess future directions for PAS in low-k films. In so doing we also want to compare strengths and weaknesses of PAS with those of the few other techniques that are being used to probe thin-film nanoporosity.

The motivation for replacing silica as the standard dielectric in microchips is simply to get around the limitations on chip speed dictated by the RC time constant of the interlayer interconnects. In its efforts to maintain the relentless pace of Moore's Law on device density and hence device speed $\mathrm{Cu}$ has replaced $\mathrm{Al}$ to reduce the resistance, $\mathrm{R}$. Capacitance, $\mathrm{C}$, has been reduced by replacing amorphous silica $(\mathrm{k}=4.2)$ with polymeric/cross linked materials with $\mathrm{k} \sim 2.8$ (called low-k dielectrics). To achieve the "ultra low-k" goal of 2.2 and below requires the addition of a fraction of a film's volume in pores (with its vacuum $\mathrm{k}$ of 1.0) so the average $\mathrm{k}$ of the two-phase porous film is reduced.

The fabrication of a porous dielectric film of virtually any porosity is not so difficult. The challenge has been, and continues to be, to make a porous dielectric film with all the necessary electrical, chemical, and mechanical properties to withstand integration in a multilevel microchip. It is easy to imagine that the pores (especially interconnected pores) may provide a ready conduit for plasma etches and ashes to produce "integration damage" such as pore collapse and/or pore filling. It is important to understand the

\footnotetext{
" Corresponding author: e-mail: Gidley@umich.edu, Phone: 734936 1134, Fax: 7347645153
} 
mechanisms of integration damage and how it is related to the fundamental pore structure of the film how does the pore size, pore interconnection length, and total porosity affect the vulnerability of the film? Monitoring these pore characteristics in $300 \mathrm{~nm}$ insulating films has been a great challenge. In bulk porous materials one simply performs traditional gas absorption - the standard of porosimetry - and observes a pressure decrease as gas absorbs into the pores. The mass of absorbed gas for a thin film on a $\mathrm{Si}$ wafer is negligible and new methods had to be developed.

2 Methods of pore characterization If one plans to use absorption porosimetry with thin films then it is necessary to devise more sensitive methods for detecting the absorption of a penetrating species. Ellipsometric porosimetry (EP) [2] uses optical ellipsometry to monitor refractive index changes incurred by the absorption of a high vapour pressure solvent (typically toluene). Similarly, X-ray porosimetry (XRP) [3] monitors the corresponding density increase for solvent penetration. These techniques are most closely allied with standard porosimetries and can produce familiar pore size distributions (PSD) as long as the pores are accessible to the solvent. Pore interconnection length is not readily discerned, nor is depth-profiling of pore structure changes easily accomplished.

X-ray and neutron scattering techniques have long existed but the minute amount of material in a thin film is again a great challenge to pore characterization. Small angle neutron scattering (SANS) [4] and X-ray scattering (SAXS) can detect pores but lose sensitivity at the nanometer scale and require either very high X-ray intensity or stacking of 10-20 films to get sufficient neutron scattering signal. A particularly useful scattering method is X-ray reflectivity (XRR) [4] at glancing angle which can depth-profile the film's electron density. When combined with a penetrating solvent this density profiling is the basis of XRP.

Positron researchers have long known that positrons and Ps can trap and monitor voids in materials. A number of spectroscopies have been developed to parameterize the annihilation process-generically called positron annihilation spectroscopy (PAS) even though the positron may be annihilating from the Ps state. The three main spectroscopies are in the time domain (how long the positron lives before annihilation), the energy of the annihilation gamma rays, and the momentum of the gamma rays (Doppler energy shifts and angular distribution). We will focus attention on recent developments in beam-based positron/Ps annihilation lifetime spectroscopy (beam-PALS or just PALS) because it has been the most informative annihilation spectroscopy in characterizing porous low-k films. We have recently written a detailed review paper [1] and the reader is referred to it for a broader perspective and more complete bibliography.

PALS is a pore/void volume characterization technique whereby the shortening of the annihilation lifetime of Ps due to collisions with the pore walls is directly correlated with the pore size. Ps in vacuum will self-annihilate into gamma-rays with a lifetime of $142 \mathrm{~ns}$, but in a pore its lifetime is shortened by the additional effect of the positron's annihilation with electrons in the pore wall material. The PALS technique has been used for some 40 years to probe the sub-nanometer voids in bulk polymers [5]. It has been demonstrated that PALS has a good calibration for the deduced pore size vs. Ps lifetime in the diameter range from $0.3 \mathrm{~nm}$ to $30 \mathrm{~nm}$ - truly a probe of nanoporous materials. In addition, the diffusion of Ps within the pores of a sample can be used to determine the length scale over which pores are interconnected - a pore interconnection length (which, in turn, provides added information on the shape of the pores). Depth-profiling can reveal inhomogeneities below the film surface from such effects as pore collapse densification or pore filling by contaminants. A reader interested in a more detailed treatment of the beam-PALS technique should see the recent review mentioned above [1] and the references therein. We will discuss the current developments in PALS low-k materials characterization, comparisons with other methods and our expectations for future development.

3 Recent progress with PALS in low-k films PALS has the attractive feature of a direct one-to one connection between fitted Ps lifetime and pore size. The quantum mechanical model first developed by Tao [6] and Eldrup [7] in the early 1980's has been extended in order to characterize both micro- and meso-pores $[8,9]$. In the past 6 years the Michigan positron group has participated in many round-robins 
where a variety of film samples are shared by different groups in order to compare pore sizes measured with different techniques. PALS agrees quite well overall with other methods as can be seen in Fig. 1. PALS tends to measure pore diameters that are slightly larger than SANS, slightly smaller than EP, and in excellent agreement with BET (standard gas absorption used by painstakingly scraping off sufficient quantities of the film for bulk analysis). We are presently working with IMEC to compare EP results with PALS for pure silica zeolite films. PALS has demonstrated resolution to its $0.55 \mathrm{~nm}$ diameter oriented channels [10] but EP may not be detecting them for lack of toluene penetration. Overall, the universal PALS pore size calibration appears to be very reliable but, as we are beginning to now hear at this conference, experiments are searching for a material specific dependence.

The discrete lifetime fitting of the lifetime

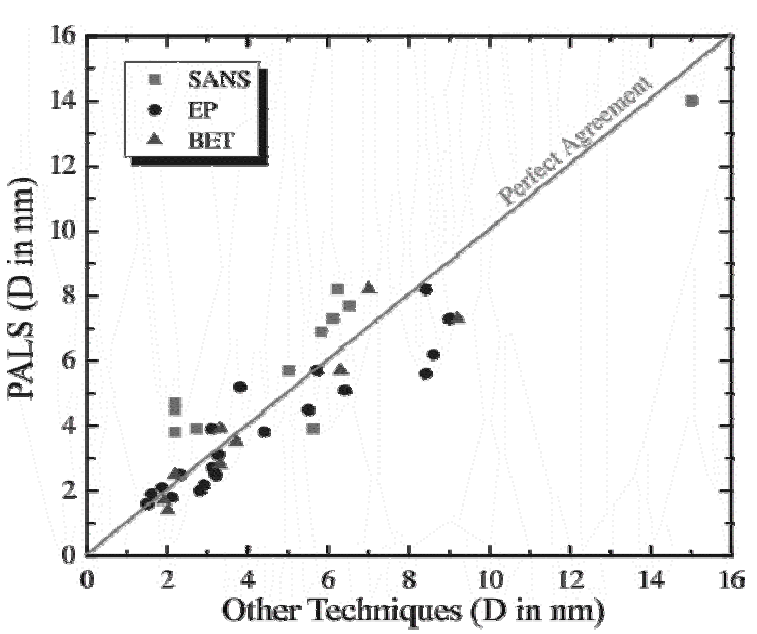

Fig. 1 Round-robin comparison of PALS-deduced pore diameter with that deduced from other techniques.

spectra is a robust procedure that determines the average size of the pores in the material. However, this analysis can be complicated by multiple-lifetime spectra that occur when micropores and mesopores coexist and each may involve interconnected pores and/or a distribution of pore sizes. To further characterize the porosity of a film, pore size distributions (PSD's) are determined by fitting the decay spectrum to a continuum of lifetimes [11] which are then converted into fractional pore volume as a function of spherical pore diameter (or cylindrical diameter). PSD results acquired at several positron beam energies are shown in Fig. 2 for a rather exotic dielectric film in which the pore structure is changing rapidly with depth below the surface [12]. One limitation of PSD [13] fitting is the uniqueness of the fitting results (a common problem in continuum fitting). Thus, the continuum fits are typically referred to as "plausible PSD's."

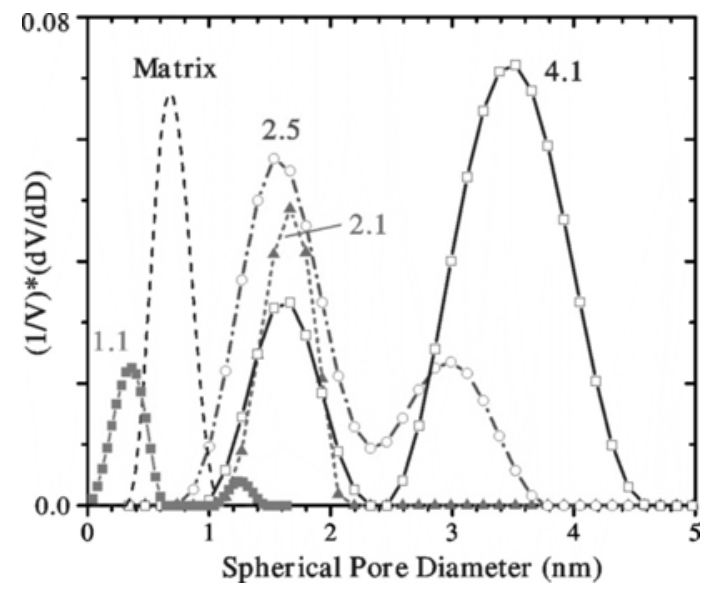

Fig. 2 Plausible pores size distributions in a low-k film as a function of positron beam energy (legend, in $\mathrm{keV}$ ) determined from continuum lifetime fitting. This film has a complicated depth-dependence to porosity.
Developing an understanding of how pores grow in films and their resulting structure is critical for the implementation of controlled pore design. PALS, with its ability to simultaneously characterize the size and interconnection length of the induced pores as a function of porosity, can be enlightening with regards to the growth of the pores in the film [14]. Porosity in films is often generated by depositing a 2-component composite where one component is a thermally degradable/sacrificial "porogen" that leaves pores behind. Porosity is adjusted by varying the porogen concentration but this also affects the aggregation of porogen domains in the matrix material. By studying the evolution of both the pore size and the pore interconnection length PALS can begin to deduce the shape of the pores and delineate the relative strength of the porogen-porogen interaction with respect to the porgen-matrix interaction. A recent study of the growth of pores in the prepercolation regime was made by using PALS on films with several cyclodextrin (CD) and calix-arene 
(CA) porogens with various functional groups in a modified MSQ host matrix [15]. Results for the pore diameter and $\mathrm{L}_{\text {int }}$ for films prepared with tCD (CD with methoxyl functional groups) SCD (CD with trimethoxyl-silyl functional groups) and CA are shown in Fig. 3. The sCD porogen system produces pores with constant Ps lifetime (hence constant mean free path), but with interconnection lengths much longer than the pore size for all porosities. As the loading fraction is increased, the pore diameter remains constant while the interconnection length continues to increase. Therefore, rather than aggregating in 3dimensions, the pores grow linearly. The trimethoxy-silyl functional groups of sCD can form Si-O-Si linkages after hydrolysis into silanol groups, and therefore covalently bonded linear chains of sCD molecules are readily formed, producing longer (more interconnected) pores of constant cross section. This growth in the cylindrical (or tubular) length is strikingly clear in Fig. 3. In the tCD system the methoxyl functional groups are known to have a weaker Van der Waals interaction, so the aggregation of the porogen domains is expected to be more 3-dimensional and random. As seen in Fig. 3 at low porosity $(\leq 8 \%)$ the pores are isolated with a diameter consistent with a template of a tCD molecule. They then gradually increase in pore size and interconnection length as the porosity is increased. This trend is consistent with uniform random population of pores in the matrix, which leads to three dimensional growth of the pores.

The CA system is unique in that PALS shows explosive growth between $7 \%$ and $15 \%$ porosity. The pore diameter jumps precipitously from a closed-pore diameter of $\sim 1.2 \mathrm{~nm}$ (consistent with the size of the templated molecule) to an effectively percolated pore with a diameter $>3 \mathrm{~nm}$. CA acts like a surfac-

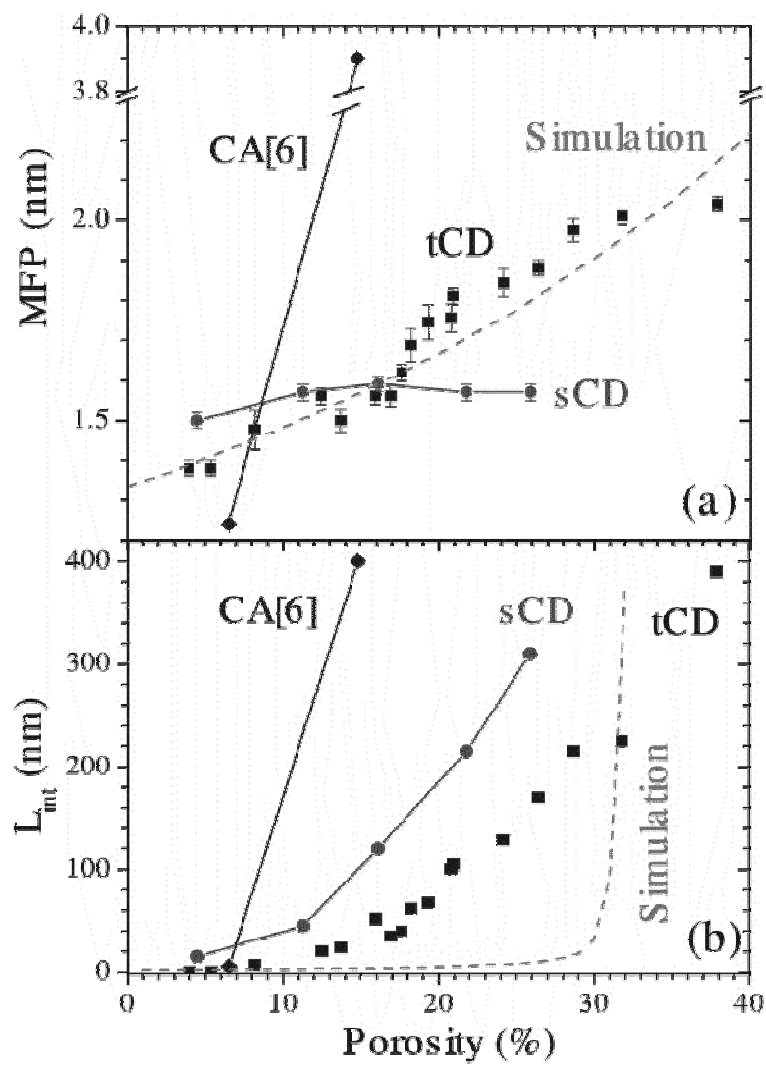

Fig. 3 Plots of pore mean free path (top) and pore interconnection length (bottom) as a function of porosity for MSSQ films made with three distinct porogens. A simulation based on random removal of cells in a cubic lattice is shown for comparison. tant, which should promote large micelle growth beyond some critical porogen concentration. In collaboration with Samsung's Advanced Institute for Technology we analyzed a finer sample step size of $3 \%$ in porosity from $7 \%$ to $22 \%$ using both EP and PALS on each film in a head-to-head comparison. At low porosity EP detects micropores of average diameter of $1.1 \mathrm{~nm}$ compared to $1.2 \mathrm{~nm}$ by PALS. At $16 \%$ film porosity the EPdeduced PSD shows an emerging peak at about $3.6 \mathrm{~nm}$ diameter. Correspondingly, PALS detects an emergent Ps lifetime corresponding to a mesopore diameter of $2.5 \mathrm{~nm}$ at $13 \%$ porosity and increases in average size to about $4 \mathrm{~nm}$ for $19 \%$ and above. In this round-robin the results of EP and PALS are so completely comparable we couldn't even claim them to be complementary. Perhaps a slight advantage is gained by PALS because we can also deduce the pore interconnection length at each film porosity. Aside from this the two methods are effectively redundant in this application.

All of these results nicely demonstrate three different porogen aggregation modes with concentration to create nanoporosity, from isolated pores to interconnected network. It is a key demonstration of the usefulness of PALS in untangling the fundamental pore structure and its evolution in porosity. Such results provide critical feedback to chemists designing the functional group chemistry that will control the pore structure. We believe this is an area that is so fundamental that further theoretical work in the form of 
simulations of pore size and interconnection length vs. porosity are warranted. Moreover, PALS in coordination with simulations may shed further light on the age-old problem of film percolation. Many traditional probes of percolation detect only the penetration of a gas or solvent through the percolated film. PALS may be a unique probe of the pre-percolation regime of finite pore size and small pore interconnection. Simulations of random pore clustering are straightforward and tend to match the pore diameter growth observed with tCD porogen above (see top panel in Fig. 3 and also Ming Liu's poster presentation at this conference). On the other hand, simulations of pore interconnection length tend not to match well with experimental measurements as shown in the bottom panel of this figure. There is a general sense in the low-k community that porogens tend to aggregate more readily than would be expected from random clustering (this feature is observed in its extreme for the $\mathrm{sCD}$ porogen above). We have been exploring different means to incorporate various schemes for porogen-porogen interactions into our simulations with limited success so far. One can easily incorporate interactions that readily shift the percolation threshold but reproducing the general (almost universal) shape of the pore interconnection length deduced by PALS remains elusive and may hold a key to deeper insight into porogen domain clustering.

4 Addressing the integration damage issue A particularly powerful feature of beam-based PALS is the ability to control the mean depth that positrons are implanted into the film by simply adjusting the energy that the incoming positrons strike the target. Depth profiling of films is a critically important aspect of beam-PALS characterization. Depth-profiling enables pore analysis of hidden layers that are inaccessible to solvents and gases and permits us to deduce pore interconnection lengths from Ps diffusion. Thus beam-based PALS can study hidden porosity beneath diffusion barriers, dense or capping layers, and multi-layer films. The diffusion barriers of any material can be tested for integrity, minimum critical thickness, and thermal stability on any underlying low-k film that has interconnected pores [16]. PALS is well suited to test diffusion barriers and advanced sealing strategies such as plasma surface densification on blanket and patterned films. Additionally, depth profiled PALS investigates inhomogeneities present in as-deposited or processed low-k films. For example, plasma densification (pore collapse) can result from exposure to plasmas in microchip processing [17, 18]. This type of "integration damage" can severely compromise the dielectric constant reduction by a low-k material [18].

A good example of the capability of PALS depth profiling was recently demonstrated in the study of a plasma-enhanced chemical vapor deposited (PECVD) SiCOH film [12]. The depth-dependent pore size distributions have already been introduced in Fig. 2 and they present indication of strongly increasing pore size and porosity with film depth. A multilayer model for the pore structure of this film that is consistent with the depth-profiled spectra is shown in the sketch of Fig. 4. (Depths in this profile are determined using the average density of the film - more (less) dense regions will be proportionately thinner (thicker) than shown). The top $30 \mathrm{~nm}$ of this film appears to be dense (not even microporous) followed by another $30 \mathrm{~nm}$ of film which is microporous only. When the film is probed more deeply, $1.5 \mathrm{~nm}$ mesopores begin to reveal themselves at depths of $60-170 \mathrm{~nm}$. Surprisingly, at depths greater than $170 \mathrm{~nm}$

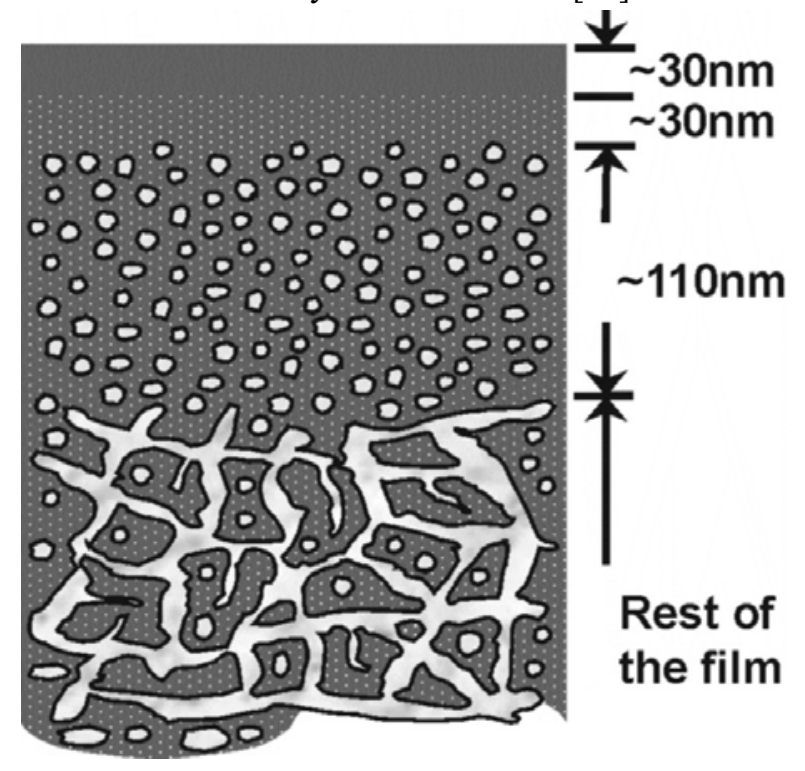

Fig. 4 Schematic of the porosity in a PECVD low-k film derived from depth profiling with PALS. 
yet another layer is discovered where $3.5 \mathrm{~nm}$ diameter pores dominate the porosity. Simple PALS depthprofiling alone cannot determine whether the large pores in this buried layer are interconnected because the telltale escape of Ps into vacuum has been curtailed by the upper layers. The solution was to etch off the upper layers to effectively move the film surface down to the layer with the $3.5 \mathrm{~nm}$ pores. PALS then confirmed [12] that these large pores are indeed highly interconnected. This layer of large, interconnected pores is precisely located where Sematech observed trench wall bowing and voids when they etched and $\mathrm{Cu}$ filled $350 \mathrm{~nm}$ deep trenches into this particular dielectric [19]. This fatal integration damage is quite certainly caused by the large, open pore structure hidden/buried below the surface of the film.

The integration challenge presented by porous dielectrics has delayed implementation of ultra low-k $(\mathrm{k}<2.2)$ dielectrics in microchips. A number of strategies are being pursued to address this challenge. A straightforward approach is to continue to fine-tune the porogen-host matrix system by adjusting the chemistry of the porogen-porgen interactions and the porogen-matrix interactions. The goal is to start with a nominally low-k matrix so requisite porosity to achieve $\mathrm{k} \sim 2.2$ is as low as possible (and well below percolation threshold). One hopes to control pore size to be less than a few nm diameter while minimizing pore interconnection length. Matrix chemistry can also influence plasma damage susceptibility. Spin-on composite films have been intensely pursued while CVD (chemical vapour deposition) films are arguably now the most popular current method being tested. An interesting twist on this fine-tuning of the matrix-porogen system is the dual porogen approach of the Seoul National University (SNU) group. In addition to the standard nanocomposite porogen generator SNU is also incorporating a second porogen that is chemically grafted onto the MSQ matrix backbone in such a concentration that roughly half of the film's mesoporosity is derived from each porogen. Our PALS results showed that this resulted in a bimodal PSD with the smallest pores corresponding to those generated by the grafted porogen (easily tested by simply removing the nanoparticle porogen). A bimodal PSD indicates that there is little interconnection between the two distinct porogen domains and, indeed, the pore interconnection length is relatively short and evidently due only to pore interconnection of the larger pores. With the clear goal to reduce pore interconnection length this novel dual porogen approach is relying on PALS analysis to judge its success.

A concomitant strategy is to attempt to seal pores near the porous dielectric surface. This can involve exposure to reactive plasmas that collapse pores preferentially at the surface [17] or by filling surface pores with a low-k material [20]. PALS is particularly useful here because it can test the integrity of the pore seal, depth-profile the surface layer, and probe the remainder of the porous film for any deep-lying damage the processing may have caused.

A totally different approach to addressing the integration challenge involves changing the integration processing itself. If the photoresist-etch process is too harsh for porous films perhaps one can side-step the most challenging problems by direct nano-imprinting trench structure into a nanocomposite with subsequent vitrification and porogen removal. Success in this process depends on successful imprint molding of the low-k dielectric without loss of significant porosity in the subsequent porogen removal. In the past year we have been working with NIST to bring together the complementary capabilities of PALS, XRR, and XRP to probe porosity, pore size, and pore interconnection of $100 \mathrm{~nm}$ features nanoimprinted in low-k MSQ-based films. Although the imprinting was successful, PALS and XRP definitively detected some loss of porosity after imprinting and the source of this loss is being investigated. At the ever-decreasing device size scale and the need for ultra-low-k ILD's we see this research in nanoimprinting low-k materials as seminal---a particularly strong future growth area for PALS research.

5 Future of PAS porosimetry is bright As a research tool for pore characterization PAS, and PALS in particular, offers many attractive porosimetry features with no apparent limitations through the $22 \mathrm{~nm}$ technology node. There is no practical limit $(\sim 0.2-0.3 \mathrm{~nm}$ diameter $)$ on the minimum pore size. Pore interconnection lengths can be deduced and pore size distributions can be fitted even if the dielectric layer is buried under a diffusion barrier. PALS has demonstrated its usefulness in characterization of multilayer films with a complex pore structure and in investigating the evolution of pore shape and struc- 
ture with porosity. Lateral resolution of several millimeters is typical as is the depth resolution of about 10 nanometers. Microbeams are available. The positron beam and spectrometer are compact, relatively uncomplicated devices with rather straightforward analysis methodology. Radiation damage to the film is negligible - the technique is non-destructive except that samples are presently cut to centimeter size from wafers. Cutting wafers and acquiring data for $30 \mathrm{~min}-1$ hour are quite acceptable nuisances in a materials research laboratory environment given the valuable information derived.

Positron researchers are continuously working to improve PALS and PAS techniques to have a broad impact in the development of future low-k films. While the current PALS technique is sufficient to support the microelectronics industry through the $22 \mathrm{~nm}$ technology node, these improvements will extend the capabilities of the technique.

Near-term improvements in PALS are expected to address three issues: positron rate, lateral resolution, and sample size. The goal is to develop the capability to perform PALS: on a $1 \mathrm{~mm}$ diameter spot; located on a full $300 \mathrm{~mm}$ wafer; in about one minute acquisition and analysis time. At this conference we have heard of many exciting developments in high intensity positron beam facilities aimed at $10^{8}-10^{10}$ slow positrons/second. We are collaborating on one such intense positron beam that is under construction at the North Carolina State University PULSTAR Nuclear Reactor in Raleigh, NC [21]. This positron beam (inspired by the intense positron facility at Delft University) is based on pair-production in the intense gamma flux near the reactor core. It is designed to include the latest generation of PALS spectrometers for depth-profiled dielectric film characterization and for nanophase characterization in general. A prototype beam has already achieved a reactor-power scaled rate of $3 \times 10^{7}$ positrons/s [21]. The full scale beam should be a factor of 10-50 higher rate, insuring more than sufficient beam to explore the maximum data rate in PALS fast-coincidence timing (limited by timing system deadtime) while focusing and limiting the beam to $\sim 1 \mathrm{~mm}$ diameter. The PALS spectrometer positron optics has been designed to test small-spot analysis on a full wafer. This university-based facility should allow fast, convenient, and inexpensive access to PALS/PAS analysis and expertise for materials researchers and engineers in both industry and academia.

The impact of PALS/PAS is not limited to low-k dielectric films. The technique has proven to be useful in studying hardmask materials, SiN and $\mathrm{SiC}$ barriers, photoresists, and should be useful in exploring a wide range of porous materials, barriers, and permeable membranes. We have heard at this conference from the Tsukuba group about exploring vacancies with PAS in high-k ultra-thin gate dielectrics [22]. As part of the continuing nanotechnology initiative we should expect to see more materials with engineered nanopores to enhance chemical, physical, or transport properties. At the true nanometer pore scale and particularly for thin films and membranes that may be difficult to access with gases or solvents PALS should become a routine analysis technique that academic and industrial researchers can reliably count on to depth profile pore size distributions and pore interconnection lengths.

Acknowledgements We would like to thank Robert Miller and Qinghuang Lin of IBM, Chris Soles of NIST, Mikhail Baklanov of IMEC, Do Yoon of Seoul National University, Richard Carter of LSI Logic (now at AMD), Todd Ryan of AMD, Jin-heong Yim of SAIT (now at Kongju University), and Ayman Hawari of NCSU for their collaborations. We thank other members of the Michigan Positron Group, Mark Skalsey and William Frieze, for dedicated support.

\section{References}

[1] D. W. Gidley, H.-G. Peng, and R. S. Vallery, Annu. Rev. Mater. Res. 36, 49 (2006).

[2] M. R. Baklanov and K. P. Mogilnikov, Microelectron. Eng. 64, 335 (2002).

[3] H. J. Lee et al., J. Appl. Phys. 95, 2355 (2004).

[4] B. D. Vogt et al., J. Phys. Chem. B 109, 18445 (2005).

[5] Y. Y. Wang et al., J. Polymer Sci. B 28, 1431 (1990).

[6] S. J. Tao, J. Chem. Phys. 56, 5499 (1972).

[7] M. Eldrup, D. Lightbody, and J. N. Sherwood, Chem. Phys. 63, 51 (1981).

[8] T. L. Dull et al., J. Phys. Chem. B 105, 4657 (2001). 
[9] T. Goworek, Radiat. Chem. Phys. 68, 331 (2003).

[10] S. Li et al., J. Phys. Chem. B 108, 11689 (2004).

[11] R. B. Gregory and Y. K. Zhu, Nucl. Instrum. Methods Phys. Res. A 290, 172 (1990).

[12] H. G. Peng, et al., Appl. Phys. Lett. 86, 121904 (2005).

[13] G. Dlubek, C. Hubner, and S. Eichler, Nucl. Instrum. Methods B 142, 191 (1998).

[14] C. L. Wang et al., Appl. Phys. Lett. 81, 4413 (2002).

[15] H.-G. Peng et al., Appl. Phys. Lett. 87, 161903 (2005).

[16] J. N. Sun et al., J. Appl. Phys. 89, 5138 (2001).

[17] J. N. Sun et al., Appl. Phys. Lett. 81, 1447 (2002).

[18] E. T. Ryan et al., Mater. Res. Soc. Symp. Proc. 766, E10.8 (2003).

[19] Y. Liu et al., Mater. Res. Soc. Symp. Proc. 863 (2005).

[20] J. S. Juneja et al., J. Vacuum Sci. Technol. B 23, 2232 (2005).

[21] A. G. Hathaway, Design and testing of a prototype slow positron beam at the NC State University PULSTAR reactor. 2005, North Carolina State University.

[22] A. Uedono et al., J. Appl. Phys. 98, 023506 (2005). 